\title{
EUSO-Balloon trigger efficiency in preparation of a long duration flight
}

\author{
Simon Bacholle*, Etienne Parizot \\ Laboratoire AstroParticule et Cosmologie - Université Paris Diderot-Paris 7, CNRS/IN2P3 \\ E-mail: bacholledapc.in2P3.fr
}

Francesco Fenu, Mario Bertaina

Dipartimento di Fisica, Università di Torino INFN Torino, Italy

\section{for the JEM-EUSO Collaboration}

\begin{abstract}
EUSO-Balloon is a pathfinder for the JEM-EUSO experiment, devoted to the observation of ultrahigh-energy cosmic rays (UHECRs) from space. It operates on a stratospheric balloon at an altitude of $\sim 38 \mathrm{~km}$. A first flight took place in August 2014, during a single night [1]. Here, we investigate the acceptance of a new version of the instrument in view of a forthcoming long duration flight. We use the ESAF simulation code [2], adapted to the EUSO-Balloon design, to determine the trigger efficiency as a function of energy. We then convolve the results with the cosmic-ray spectrum and derive the number of events expected to be detected in the range between $10^{17.5} \mathrm{eV}$ and $10^{19} \mathrm{eV}$. We conclude that EUSO-Balloon is well-designed to be the first fluorescence telescope to detect cosmic-ray showers from above.
\end{abstract}

The 34th International Cosmic Ray Conference,

30 July- 6 August, 2015

The Hague, The Netherlands

${ }^{*}$ Speaker. 


\section{Introduction}

The EUSO-Balloon instrument has been conceived as a prototype to the space mission JEMEUSO [3], dedicated to the study of ultra-high-energy cosmic rays (UHECRs) from space, through the detection of the fluorescence emission induced by the resulting atmospheric showers. EUSOBalloon's main instrument consists of a UV telescope with Fresnel optics giving a field of view of $\pm 5.5^{\circ}$, and a focal surface equipped with 36 multi-anode photomultiplier tubes (MAPMT) of 64 pixels each, with single photon sensitivity. The instrument is designed to fly at $\sim 38 \mathrm{~km}$ altitude. The first flight of EUSO-Balloon took place in the night of $24^{\text {th }}-25^{\text {th }}$ august 2015 , as a mission of the French national space agency, CNES, with contributions of the international JEMEUSO collaboration for various subsystems (see [1] for details about the mission, the flight and the instrument performances).

Given the short flying time and EUSO-Balloon's energy threshold for cosmic-ray shower detection around $10^{18} \mathrm{eV}$, no actual cosmic-ray detection was expected. To remedy the absence of such interesting physical events, a helicopter was flown under the balloon for a period of $\sim 2$ hours, flashing intense light sources and shooting horizontal laser beams. The scattered light of these laser shots was indeed detected by the EUSO-Balloon telescope and their tracks have been reconstructed (see [4], [5] and [6]). The next crucial step of this pathfinder program to JEM-EUSO will be the detection of the air fluorescence light of genuine cosmic-ray (CR) air showers from above for the first time. The EUSO-Balloon detectors should be capable of do so, provided that the instrument trigger efficiency will provide enough statistics, taking into account the UHECR flux and the instrument exposure.

In this paper, we investigate the acceptance of the EUSO-Balloon detector as a function of energy, in preparation of a long duration flight which could occur in 2017 [7]. At this time, we implement the expected EUSO-Balloon's performances and the UV background measurements derived from the first flight into the simulation code ESAF [refs.], and simulated cosmic ray showers with different energies, arrival directions and positions with respect to the instrument. We only simulate protonic showers for this study. This is a rather conservative choice, since the trigger efficiency for heavier nuclei is higher, because of the higher shower development in the atmosphere (on average).

\section{The simulations}

The ESAF simulation code includes four distinct parts: i) the simulation of cosmic showers of a given energy with a given geometry, ii) the transport of the photons generated by the shower (both air fluorescence and Cherenkov) up to the pupil of the instrument, iii) the propagation of the incoming photons through the optical system up to the focal surface, iv) the detection of the photons by the MAPMTs and the resulting generation of the electronics signal (see [2] for more details). In addition, ESAF can simulate a random UV background with a given total intensity, expressed in $\mathrm{ph} / \mathrm{m}^{2} / \mathrm{sr} / \mathrm{ns}$. Finally, a specific trigger algorithm that was developed to detect and identify shower tracks [8] has been implemented. In this algorithm, referred to as Persitent Track Trigger (PTT), pixels are grouped in $3 \times 3$ boxes. If, during a fixed number of frames, the signal in 


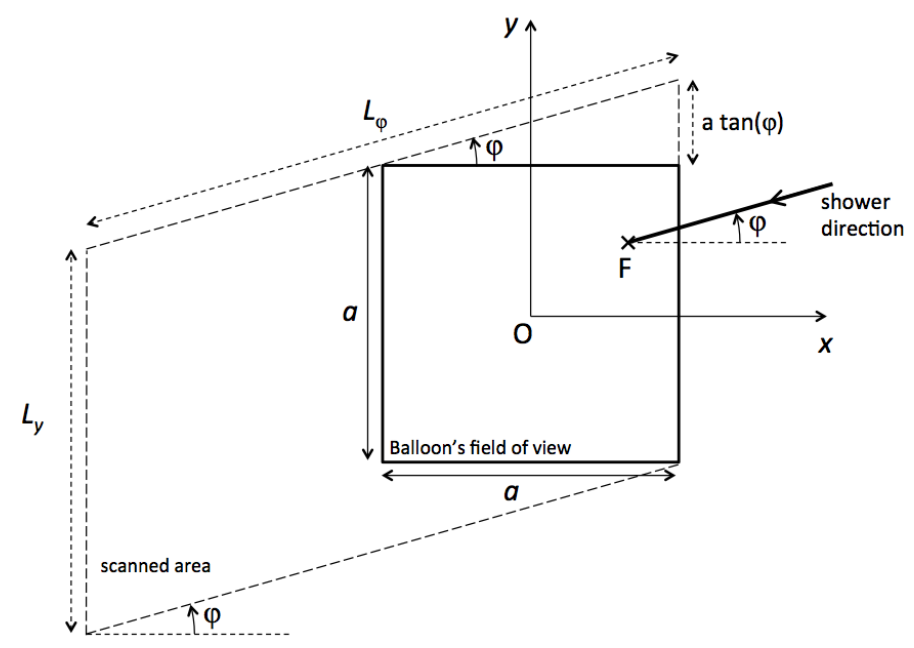

Figure 1: Schematic view of the scanning strategy and axis conventions (see text).

at least one pixel of the box reaches a preset threshold $n_{t h r}^{p i x}$, and the total signal in the box is higher than another preset threshold $n_{t h r}^{b o x}$, a trigger is issued.

The instrument has a square entrance pupil of area $S=1 \mathrm{~m}^{2}$. The optics has an opening angle $\alpha \simeq 11^{\circ}$, so that the field-of-view (FoV) projects on the ground as a square of a side $a=$ $2 H \tan (\alpha / 2)$, where $H$ is the altitude of the balloon. For $H=38.5 \mathrm{~km}, a \simeq 7.5 \mathrm{~km}$. The photon detectors are MAPMTs covered with BG3 UV filters (with high transparency in the 300-400 nm range). The total optics throughput and the photo-detection efficiency (including collection and quantum efficiencies) are treated globally through a dimensionless parameter which can be adjusted to investigate the impact of a possible degradation of the instrument. We assume a nominal total efficiency of 0.60 (optics) $\times 0.20$ (PMT) $=0.12$.

\section{Trigger efficiency and effective detection surface}

\subsection{Strategy}

Our main goal is to characterize the trigger efficiency of the instrument as a function of shower direction, shower foot position (the intersection of its axis with the ground), and energy. To achieve this goal, we simulate the showers with different characteristics, according to the following procedure.

First, we take advantage of the symmetries. We use the geometry represented in Fig. 1, with the origin $(x=0, y=0)$ at the nadir of the balloon, and the $x$-axis and $y$-axis along the sides of the FoV. The zenith and azimuthal angles of the shower are noted as $\theta$ and $\varphi$, respectively. The shower triggering efficiency as a function of azimuthal angle, $\varphi$, has a rotational symmetry of angle $\pi / 2$. In addition, the trigger efficiency is symmetric with respect to the $x$-axis. Therefore, we can restrict the exploration of the azimuthal dependence of the trigger efficiency to the range of $0^{\circ} \leq \varphi \leq 45^{\circ}$.

In order for a CR shower to trigger the instrument, it is not necessary that its foot be located inside the FoV of the telescope. A shower with its maximum within the FoV will hit the ground the 
further away from the balloon's nadir the larger zenith angle it has. To make sure that we consider all the showers that can trigger the instrument, we scan over a larger area around the nominal FoV, where we force the simulated shower to hit the ground. We start with the shower foot positions on the rightmost line where showers could possibly trigger the detector, namely, the line $x=a / 2$. Then we move towards smaller values of $x$ along the azimuthal direction (so-called $\varphi$-axis), until the shower foot, F, reaches the limit beyond which the shower track does not pass through the FoV at all. This limit depends on the zenith angle $\theta$. The resulting scanning area is represented on Fig. 1 by the inclined parallelogram. Its length along the $y$-axis is $L_{y}=a(1+\tan \varphi)$. For the length along the $\varphi$-axis, $L_{\varphi}$, we chose a smaller value than imposed by geometry alone, recognising that, in practice, not only some part of the shower track should be located in the FoV, but also a relatively small region around the shower maximum. We, thus, used the following empirical value: $L_{\varphi}=a+h_{0} \times \tan \theta$, with $h_{0}=12 \mathrm{~km}$.

Once the scanning area is defined, we simulate showers, with feet at positions $\left(x_{i}, y_{j}\right)$, where $i$ and $j$ run from 0 to $N-1$, and:

$$
x_{i}=\frac{a}{2}-i \times \frac{L_{\varphi} \cos \varphi}{N-1}
$$

and

$$
y_{j}=\frac{a}{2}+\left(x-\frac{a}{2}\right) \tan \varphi+j \times \frac{L_{y}}{N-1},
$$

We simulate $N \times N$ showers in total for each detector configuration and each shower parameter set. We chose $N=100$, and simply keep track of the foot positions of all the showers which did trigger the detector.

\subsection{Results}

Figure 2 shows the results obtained for CR showers with $\theta=45^{\circ}$ and $\varphi=0^{\circ}$, with energies from $10^{18.0} \mathrm{eV}$ to $10^{18.5} \mathrm{eV}$, with the instrument's altitude $H=38.5 \mathrm{~km}$, and the UV background set to $B=300$ photons $/ \mathrm{m}^{2} / \mathrm{sr} / \mathrm{ns}$. The feet of the simulated showers that did trigger the detector appear as heavy red circles, while those of the showers that did not trigger are in a light grey color. The projected FoV on ground is represented by a dashed rectangle (which is a square of side $a \simeq 7.3 \mathrm{~km}$ for the altitude of $38 \mathrm{~km}$ ).

As expected, the number of triggering events, $N_{\text {trigg }}$ is seen to be an increasing function of energy (for a fixed arrival direction). It allows us to determine the trigger efficiency, which is essentially zero below $10^{17.5} \mathrm{eV}$, and $100 \%$ above $10^{18.5} \mathrm{eV}$, for the showers with the shower maximum position, in the FoV of the instrument. However, because the fluorescence light is emitted over the extended track, showers with higher energy can trigger the detector over a larger surface than the projected FoV. To determine the effective aperture of the detector at a given energy, the critical quantity is the total area over which a shower developing along a given direction can be detected, weighted by the trigger efficiency. This may be called the effective area of the detector, $S_{\text {eff }}(E, \theta, \phi)$.

Each shower foot position on the ground associated with a triggering shower may be constructed as adding an elementary surface $\Delta S=\Delta x \times \Delta y$ to the effective detector area, where

$$
\Delta x=\frac{L_{\varphi} \cos \varphi}{N-1} \quad \text { and } \quad \Delta y=\frac{L_{y}}{N-1} .
$$



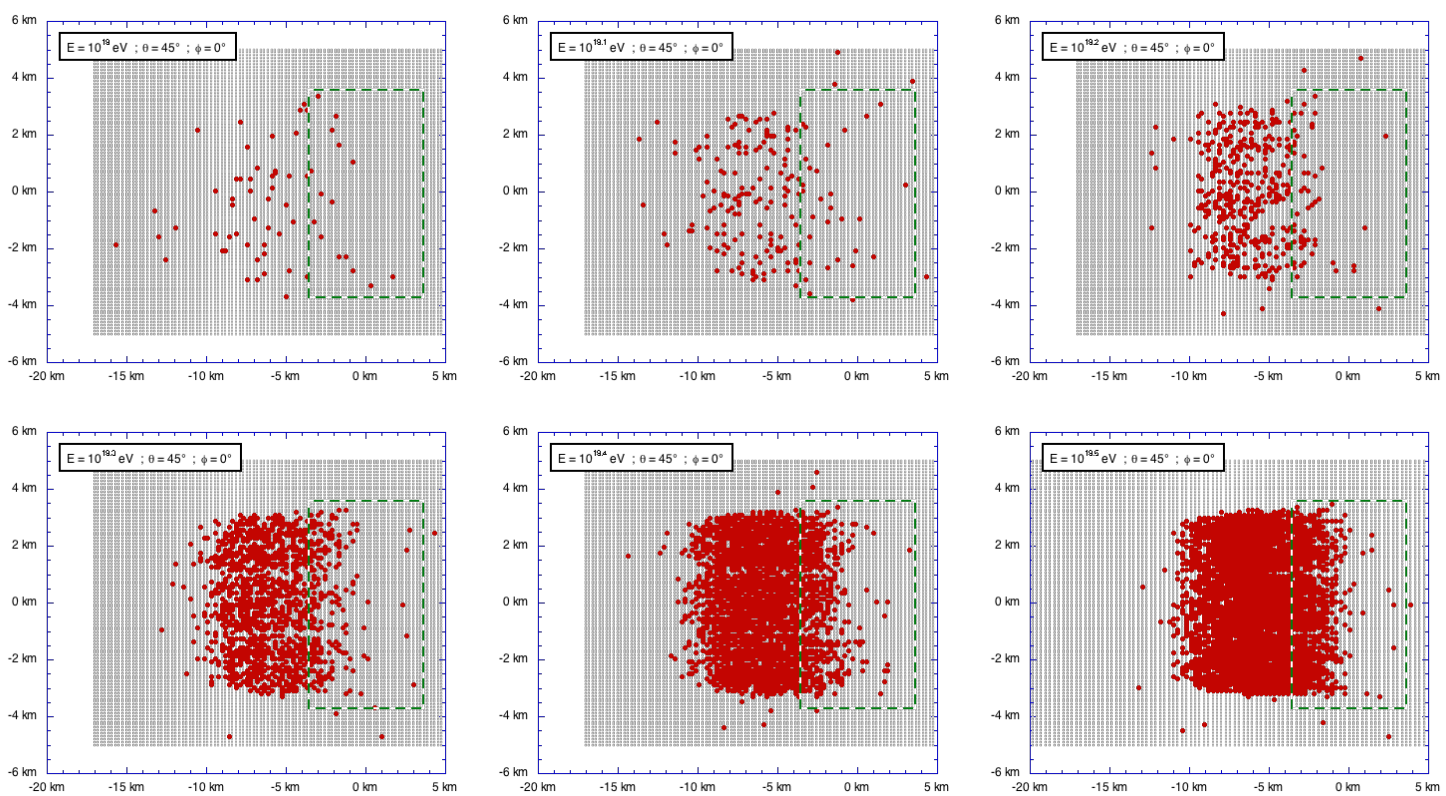

Figure 2: Shower foot position of the triggering showers (in red) and non triggering showers (in light grey), for CR showers with zenith angle $\theta=45^{\circ}$, azimuth $\varphi=0^{\circ}$, and energies going from $E=10^{18.0} \mathrm{eV}$ (top left) to $E=10^{18.5} \mathrm{eV}$ (bottom right), with increments of $0.1 \mathrm{dec}$. The dashed lines represent the camera's field of view.
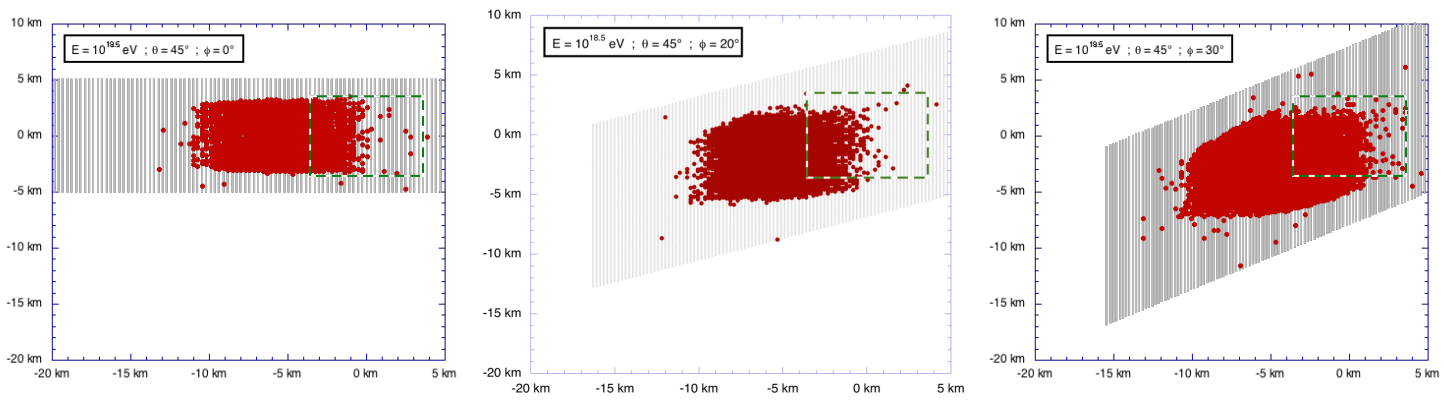

Figure 3: Same as Fig. 2, for showers with $E=10^{18.5} \mathrm{eV}$, with azimuthal angles $\varphi=0^{\circ}, 20^{\circ}$ and $30^{\circ}$.

The total effective area for the showers of a given energy and arrival direction is, thus, simply obtained as:

$$
\Delta S_{\text {eff }}(E, \theta, \varphi)=N_{\text {trigg }} \times \Delta x \times \Delta y .
$$

In Fig. 3, we show the azimuthal dependence of the shower foot positions of the triggered events, for CRs with $E=10^{18.5} \mathrm{eV}$, zenith angle $\theta=45^{\circ}$, and azimuth $\phi=0^{\circ}, 20^{\circ}$ and $30^{\circ}$ (from left to right). At such a high energy, where the local detection efficiencies are close to 1 , the effective detection area is the horizontal cross-section of the instrument's FOV at the shower maximum projected on the ground along the shower axis.

In Fig. 4, we show the evolution of $S_{\text {eff }}$ as a function of energy (left), azimuth (center) and 

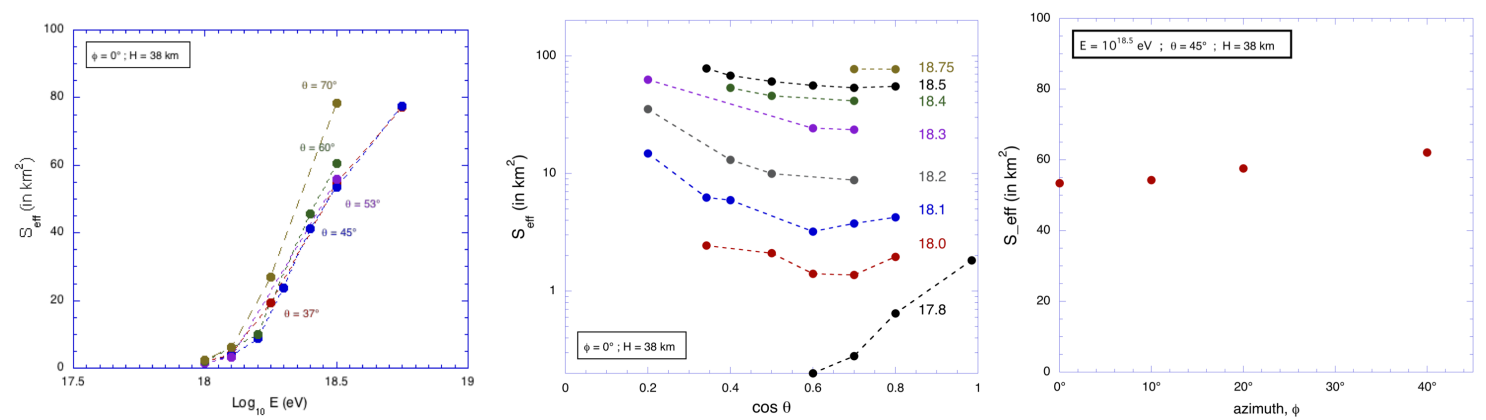

Figure 4: Effective detection surface as a function of energy for showers with $\varphi=0^{\circ}$ and different zenith angles (left), as a function of zenith angle for showers with $\varphi=0^{\circ}$ and different energies (center), and as a function of azimuth, for showers with $E=10^{18.5} \mathrm{eV}$ and $\theta=45^{\circ}$.

zenith angle (right). The detecting power of the instrument is a strongly increasing function of energy above $\sim 10^{18} \mathrm{eV}$, as was already visible in Fig. 2. Not surprisingly, the azimuthal dependence of the effective detection area is rather weak (it would be inexistent if the detector's focal surface was not square and had the same rotational symmetry as the lenses).

The influence of the UV background on the trigger efficiency has been intensively studied in earlier works (see [8], p. 134). An increase of this background by a factor $\alpha$ results in a global shift of the acceptance evolution curve towards higher energies, by a factor $\sqrt{\alpha}$. During the flight of EUSO-Balloon, an increase by a factor $\sim 1.6$ was measured when the instrument flew over thick clouds because of their higher capacity to reflect UV light. A higher moon phase also results in a larger background.

We also studied the effective detection surfaces obtained for a balloon altitude of $H=30 \mathrm{~km}$. The preliminary results shows that the resulting energy threshold is lowered by $\sim 25 \%$ (global shift of the acceptance curve).

\section{Estimates of the acceptance and event rate}

Combining the effective detection surfaces at different zenith and azimuthal angles, one can obtain the aperture of the instrument in $\mathrm{km}^{2} \mathrm{sr}$ as a function of energy (for a given background $B$ and altitude $H$ ):

$$
\mathscr{A}(E ; B, H)=\iint S_{\mathrm{eff}}(E, \theta, \varphi ; B, H) \cos \theta \mathrm{d} \Omega=\int_{\theta=0}^{\pi / 2} \int_{\varphi=0}^{2 \pi} S_{\mathrm{eff}}(E, \theta, \varphi ; B, H) \cos \theta \sin \theta \mathrm{d} \theta \mathrm{d} \varphi .
$$

Defining the quantity $\Sigma_{\text {eff }} \equiv 2 \pi\left\langle S_{\text {eff }}\right\rangle \cos \theta$, where $\left\langle S_{\text {eff }}\right\rangle$ is the average of $S_{\text {eff }}$ over azimuthal angles, the acceptance can be rewritten (dropping the implied parameters) as:

$$
\mathscr{A}(E)=\int_{0}^{1} \Sigma(E, \cos \theta) \mathrm{d} \cos \theta .
$$

This quantity, which may be called the differential acceptance, represents the effective acceptance for a cosmic-ray shower detection "per unit of $\cos \theta$ ". It also gives the expected distribution 

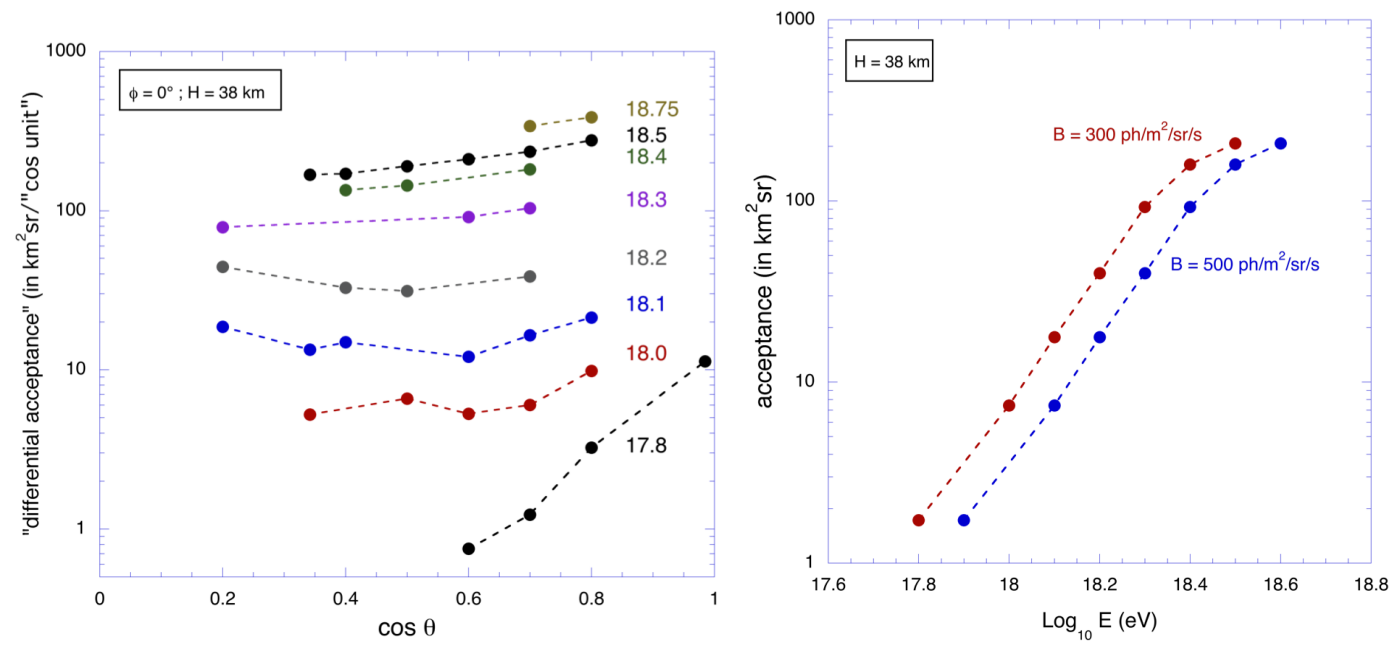

Figure 5: Left: Estimated differential acceptance (see text), as a function of zenith angle cosine, for different energies. Right: resulting acceptance as a function of energy, in $\mathrm{km}^{2} \mathrm{sr}$ for two different background levels.

of events as a function of the zenith angle. Its integral over $\cos \theta$ gives the total acceptance of the detector. The result is shown in Fig. 5. Apart from the obvious global increase with energy, it is interesting to note that the zenith angle distribution of the detected showers depends on energy. For low energy showers, close to the detection threshold, only vertical or close to vertical showers can be observed. This is because more inclined ones will go through a larger depth of atmosphere, and will, thus, have "died" before entering the field of view of the instrument.

We can then in principle obtain the total exposure of the sky for a given flight of the instrument, by integrating the aperture over time, taking into account the instantaneous background level, $B(t)$, and altitude, $H(t)$, as well as the periods when shower detection is impossible (city lights, highaltitude clouds, etc.):

$$
\mathscr{E}_{\mathrm{tot}}(E)=\int_{\text {flight }} \mathscr{A}(E ; B(t), H(t)) \delta_{\mathrm{op}}(t) \mathrm{d} t
$$

where $\delta_{\mathrm{op}}(t)$ is a function describing the operational status (on/off) of the instrument at time $t$.

Finally, we can estimate the number of cosmic-ray events which may be expected to be detected during the next EUSO-Balloon's flight by the exposure accumulated during the flight:

$$
N_{\mathrm{CR}}=\int \mathscr{E}(E) \times \Phi(E) \mathrm{d} E
$$

where $\Phi(E)$ is the differential cosmic ray flux, in $\mathrm{eV}^{-1} \mathrm{sr}^{-1} \mathrm{~s}^{-1}$.

For this estimate, we use an average spectrum based on the Auger measurements ([9]). The resulting number of events is $\sim 0.15$ per hour of data taking with a nominal background $B=$ $300 \mathrm{ph} / \mathrm{m}^{2} / \mathrm{sr} / \mathrm{ns}$ at an altitude of $38 \mathrm{~km}$, i.e. $\sim 1.2$ events per 8-hour night, and $\geq 15$ events for a three-weeks flight with nights of $\sim 8$ hours, taking into account a reduction of the efficiency at larger moon phases. 
This estimate is subject to a rather large uncertainty, due to the various uncertainties relating to the background levels over the areas that will be overflown by the balloon and to the pioneering nature of the mission. Therefore, we chose to adopt rather conservative assumptions, notably as far as the global optics and photo-detector efficiencies are concerned. Here, we assumed an overall efficiency of $12 \%$, which results from $20 \%$ efficiency for the focal surface and $60 \%$ for the optics throughput. The average photomultiplier tubes are expected to have a higher efficiency. The actual detection efficiency of the instrument may be higher at all energies. Given that the CR flux is a strongly decreasing function of energy, a lower energy threshold may result in larger numbers of detected events. Likewise, we assumed the highest floating altitude for the balloon, while a lower altitude will also result in a lower energy threshold and larger detection rate. Preliminary simulations (for showers with zenith angles between $37^{\circ}$ and $67^{\circ}$ only), show that the aperture of the instrument is typically increased by a factor of order 2 (up to 2.3) at energies between $10^{17.8} \mathrm{eV}$ and $10^{18.2} \mathrm{eV}$, where most events are expected to be detected.

We believe that the estimated event rate gives a good confidence that EUSO-Balloon's long duration flight will indeed provide the first detection and measurement of the air fluorescence light of the CR shower from above, and, thus, mark an important milestone in the development of the space road to high-aperture UHECR detectors.

\section{References}

[1] P. von Ballmoos, General overview of EUSO-Balloon mission, in proceedings of the 34th ICRC, POS ( ICRC2015) 0725.

[2] C. Berat et al, ESAF: Full Simulation of Space-Based Extensive Air Showers Detectors, Astropart. Phys. 33 221-247.

[3] M. Bertaina,; E. Parizot, The JEM-EUSO mission: a space observatory to study the origin of Ultra-High Energy Cosmic Rays, Nucl. Phys. B Proc. Suppl, 256 275-286. [arxiv:1501.06368v2].

[4] J. Eser, Laser direction reconstruction in EUSO-Balloon experiment, in proceedings of the 34th ICRC, POS ( ICRC2015) 0860.

[5] A.Jung, Search for significant background variations in the EUSO-Balloon data, in proceedings of the 34th ICRC, POs ( ICRC2015) 0971.

[6] G. Suino, Tests of JEM-EUSO 1st level trigger using EUSO-Balloon data, in proceedings of the 34th ICRC, POS ( ICRC2015) 0925.

[7] L. Wiencke, EUSO-Balloon mission to record extensive air showers from near space, in proceedings of the 34th ICRC, posPos(ICRC2015)0816.

[8] F. Fenu, A Simulation Study of the JEM-EUSO Mission for the Detection of Ultra-High Energy Cosmic Rays, PhD thesis.

[9] A.Schulz, The measurement of the energy spectrum of cosmic rays above $3 \times 10^{17} \mathrm{eV}$ with the Pierre Auger Observatory, in proceedings of the 33rd ICRC. 